\title{
Spots on Betelgeuse, what are they?
}

\author{
Andrea K. Dupree ${ }^{1}$ \\ ${ }^{1}$ Smithsonian Astrophysical Observatory/Harvard-Smithsonian Center for Astrophysics \\ 60 Garden Street, Cambridge, MA 02138 USA \\ email: dupree@cfa.harvard.edu
}

\begin{abstract}
The supergiant star Alpha Orionis (Betelgeuse) is the only star other than the Sun to be spatially resolved either through direct imaging or through reconstruction of interferometric observations. Centimeter-radio wavelength, infrared and ultraviolet images reveal a few bright hot spots in the photosphere and chromosphere that possess characteristics different from sunspots. Large photospheric spots on Betelgeuse appear to result from convective motions, consistent with radiative hydrodynamic modeling; the chromospheric hot spots may be produced by shock waves in the chromosphere excited by the convective motions or pulsation in the photosphere. Bright chromospheric spots that cluster around the pole of Betelgeuse could be a natural result of shock breakout in a rotating star.
\end{abstract}

Keywords. stars: supergiants, stars: imaging

\section{Introduction}

The helium-burning red supergiant Betelgeuse ( $\alpha$ Orionis; HD 38901) is a bright star in the sky, large in apparent size ( $\sim 50$ mas angular diameter in the optical), and hence a favorite target for numerous observational programs and numerical simulations. In fact, over 460 papers discussing Betelgeuse have appeared in the refereed literature over the past 20 years. The star is massive, currently $\sim 18 \mathrm{M}_{\odot}$, large $\left(\sim 950 \mathrm{R}_{\odot}\right)$, with luminosity $\sim 10^{5} \mathrm{~L}_{\odot}$, and cool, $\mathrm{T}_{\text {eff }}=3650 \mathrm{~K}$ (Harper et al. 2008 ). It is classified as a semi-regular pulsating star of spectral type M2Iab. In contrast to the low $\mathrm{T}_{\text {eff }}$, chromospheric emission in the form of $\mathrm{Mg}$ II is observed, but higher ion species such as C IV, O VI, and Xrays are absent (Dupree et al. 2005) suggesting that temperatures in excess of $\sim 10000 \mathrm{~K}$ do not exist. Centimeter-wave radio observations indicate the presence of much cool material (Lim et al. 1998; Harper \& Brown 2003) coexisting with the warm chromospheric component. The star possesses a massive wind amounting to $\sim 10^{-5} \mathrm{M}_{\odot} \mathrm{yr}^{-1}$. This rate exceeds the solar wind by a factor of $10^{9}(!)$ at least, and it is not known definitively what drives the wind. Dust is formed at a great distance $\left(\sim 30 \mathrm{R}_{\star}\right)$ and has no effect on the atmospheric extension or acceleration of the warm chromosphere. Most recently, a weak longitudinal magnetic field of $\sim 1 \mathrm{G}$ has been detected through the measurement of the Stokes V parameter (Auriére et al. 2010). Ultraviolet monitoring of the total flux from Betelgeuse provided the first evidence that its light behavior and 'spottedness' are quite different from the signals of magnetic activity found in the Sun and active cool stars. In subsequent sections, the appearance of the photosphere and chromosphere from spatially resolved observations are discussed as well as our current understanding of the processes producing these phenomena.

\section{Spatially unresolved ultraviolet observations}

Quantitative studies of the Betelgeuse atmosphere started in earnest with synoptic spectra from the International Ultraviolet Explorer (IUE) satellite in conjunction with 

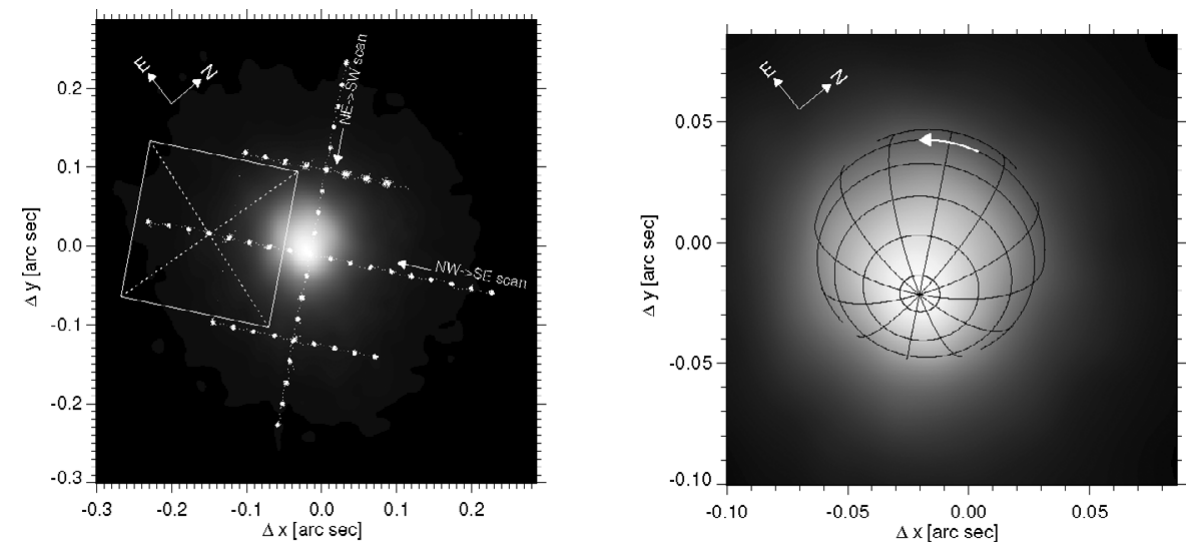

Figure 1. Alpha Ori observations with the FOC and GHRS: Left panel: Positions of the GHRS aperture (200 mas square) placed across the ultraviolet image (Gilliland \& Dupree 1996) indicating position and direction of spectral scans. Right panel: Wire frame model of Betelgeuse overlaid on the ultraviolet image (JD.)The arrow indicates the direction of rotation. The bright spot appears at the rotation pole of the star. From Uitenbroek et al. (1998).

ground-based optical photometry. These measurements of course have no spatial resolution and monitor the radiation from the whole star. Several indications of the physical processes in the Betelgeuse atmosphere emerged from the IUE studies (Dupree et al. 1987). First, the continuum brightenings and chromospheric (Mg II) brightenings are correlated which is opposite to the behavior of magnetically active stars where dark spots cause diminution of continuum light coincident with chromospheric brightenings. The amplitude of this flux modulation (about a factor of two) exceeds that found in other low gravity stars. Secondly, a photometric period of 420 days lasted for 3 years (1984$1986)$, and was obvious in the optical $(\lambda 4530)$ and ultraviolet $(\lambda 3000)$ continua as well as the Mg II emission cores. If the period of 420 days results from rotation of the star, a rotational velocity is demanded which exceeds the observed photospheric line widths. The fundamental mode of pulsation for a star like Betelgeuse (Stothers \& Leung 1971; Lovy et al. 1984) is approximately 400 days. Radial velocity variations with this period were also found (Smith et al. 1989). Thirdly, a lag occurs between initial variations in the optical and ultraviolet continua and the Mg II h emission, and subsequently the $\mathrm{Mg}$ II k line emission. These quantities are formed in successively higher atmospheric layers, and the sequential variation suggests the presence of a travelling disturbance. The 420-day variability disappeared in the years that followed (Dupree et al. 2010) confirming the 'semi-regular' classification of the supergiant. In sum, more than two decades ago, it was clear that while Betelgeuse varied in radiation output, the star did not present a classical case of magnetic sunspot/starspot variability found in the Sun or active cool stars. And, pulsation appeared to be a likely candidate for the variability.

\section{Spatially resolved ultraviolet imaging}

The advent of the Hubble Space Telescope (HST) and its Faint Object Camera (FOC) with spatial sampling of 14.35 mas per pixel made a direct image of Betelgeuse possible in the near-ultraviolet continuum $\lambda 2550$ (Fig. 1). The ultraviolet diameter of the star turned out to be about 2.2 times larger at $108 \pm 4$ mas than the optical diameter (Gilliland \& Dupree 1996) directly indicating an extended chromosphere. Most dramatically, one unresolved bright spot appeared on the disk. It occupied $10 \%$ of the area and produced 
$20 \%$ of the ultraviolet flux from the star. The spot is hot and at least $200 \mathrm{~K}$ or more, warmer than the surrounding material at $5000 \mathrm{~K}$ in the low chromosphere which forms the ultraviolet continuum at $\lambda 2550$.

Spectra in the UV were obtained with the GHRS instrument on HST and revealed the rotation of the star as shown in Fig. 1. The chromospheric hot spot appears to be located over the pole of the star. Uitenbroek et al. (1998) find a rotation period of 17 years based on an optical radius of $770 \mathrm{R}_{\odot}$. A more recent estimate of the radius of Betelgeuse of $950 \mathrm{R}_{\odot}$ (Harper et al. 2008) leads to a longer period of $21 \mathrm{yr}$. Yet both periods appear short for a large evolved star, although not out of the question for certain scenarios (see discussion in Uitenbroek et al. 1998).

Subsequent imaging with the FOC (Dupree et al. 2010) spanning a period of $\sim 4 \mathrm{yr}$ shows arc-like brightenings at times, located near or around the pole, and the spots number at most $\sim 3$ on the ultraviolet surface (Lobel \& Dupree 2000; Dupree et al. 2010)
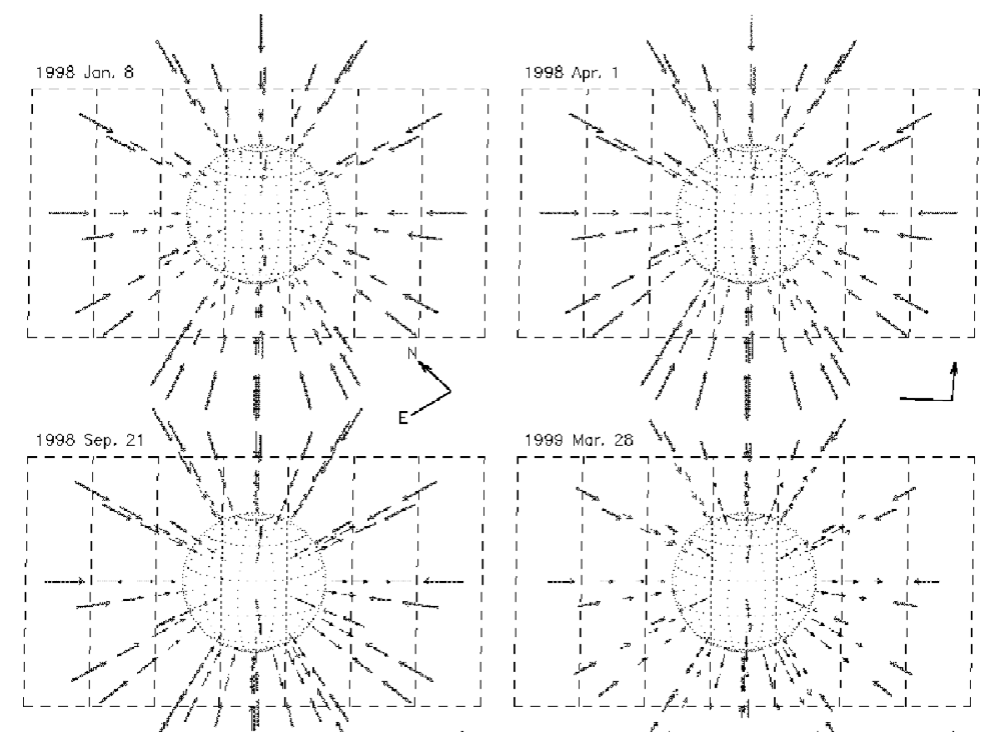

Figure 2. Velocity fields in the low chromosphere of Betelgeuse at 4 successive times. Arrows indicate the direction and magnitude of the material flow as inferred from measurements and semi-empirical non-LTE models of the Si I line profile (From: Lobel \& Dupree 2001).

\section{Spatially resolved UV spectroscopy: chromospheric motions}

Spatially resolved UV spectroscopy with the STIS slit $(25 \times 100$ mas $)$ revealed the dynamics of the supergiant's atmosphere (Lobel \& Dupree 2001). Ultraviolet spectra were obtained using a scanning motion in several directions across the extended stellar image of Betelgeuse. The raster pattern was executed 4 times over a time span of 15 months. The Si I $\lambda 2516$ line was measured and found to display varying asymmetries with position and in time; these profiles indicate the presence of outflows and inflows of material. The Si I line was modeled in non-LTE formulation which included the presence of velocity fields. Fig. 2 shows a three-dimensional representation of the motions in the extended chromosphere. The global downflow observed over the first four months reverses into subsonic outflow and this outflow is enhanced in the last observation, 15 months after the first. Clearly nonradial movements of the chromosphere are present in confined regions as a part of the general chromospheric oscillations. The upper chromosphere, as 

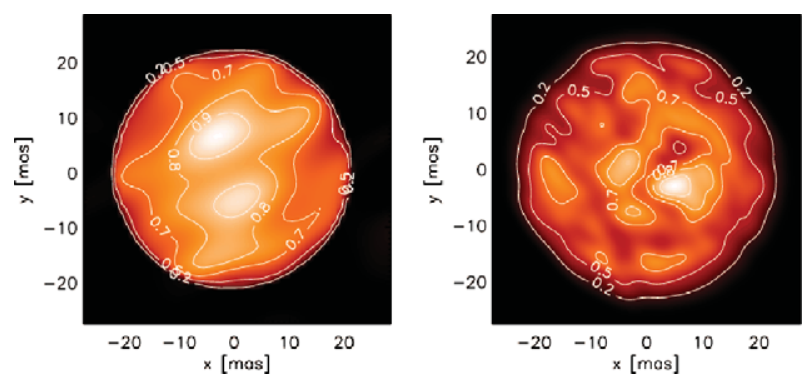

Figure 3. IR observations and simulation from Chiavassa et al. (2010). Left panel: H-band image of photosphere obtained with IOTA reconstructed from Haubois et al. (2009) showing the presence of two bright spots. Right panel: Snapshot from the 3D radiative hydrodynamic simulations of Chiavassa et al. (2010) which best matches the H-band image.

probed with the Mg II k line (formed higher in the atmosphere) shows a clear outflow when profiles sample the extended disk (Uitenbroek et al. 1998). Thus a warm $(\sim 8000 \mathrm{~K})$ wind emanates from the chromosphere.

\section{Interferometric observations}

Centimeter-wave observations of Betelgeuse with the VLA also detected one bright spot in the chromosphere (Lim et al. 1998; Harper \& Brown 2003) which is believed to have enhanced temperature as well (Harper et al. 2001). These radio observations probe the cool component of the chromosphere $(\sim 3800 \mathrm{~K})$ but no velocity measures are possible from the continuous emission.

Interferometers have recently achieved phase closure in the infrared allowing spatially resolved imaging. Ohnaka et al. (2009) imaged Betelgeuse using AMBER with the VLTI in the K-band and also lines of CO in January 2008. At the time of the observations, the photosphere as measured in K-band appeared as a uniform limb-darkened disk, but the CO lines, formed higher up in the atmosphere show inhomogeneous structures across the surface, and differing velocity patterns of inflow and outflow with amplitudes of $10-15 \mathrm{~km} \mathrm{~s}^{-1}$. AO narrow-band imaging with the VLT/NACO instrument between 1.04$2.17 \mu \mathrm{m}$ was carried out in January 2008 (Kervella et al. 2009). Selection of good images (the 'lucky' approach) revealed an asymmetric envelope and a bright plume extending out to 6 stellar radii, possibly originating from the pole of the star. These observations again document the asymmetric irregular outer atmosphere of Betelgeuse as found earlier in H- $\alpha$ speckle images (Hebden et al. 1986). H-band $(1.86 \mu \mathrm{m})$ interferometry with IOTA in October 2005 revealed two bright spots on the temperature-minimum surface of Betelgeuse (Haubois et al. 2009). These infrared measures probe the surface structures in the photosphere where convective motions are thought to occur.

\section{Hydrodynamic modeling of betelgeuse: star in a box}

Simulations of whole star convection ('Star in a Box') appear to confirm the presence and scale of the large convective cells in the photosphere of Betelgeuse. Simulations of time sequences of a supergiant are used to generate intensity maps from which the parameters measured by interferometry (visibility amplitudes and phases) are constructed (Chiavassa et al. 2009, 2010). These simulations give reasonable agreement with the interferometric results (Fig. 3) producing a few bright cells on the stellar surface. An attempt (Steffen \& Freytag 2007) to evaluate the appearance of convection in a rotating 


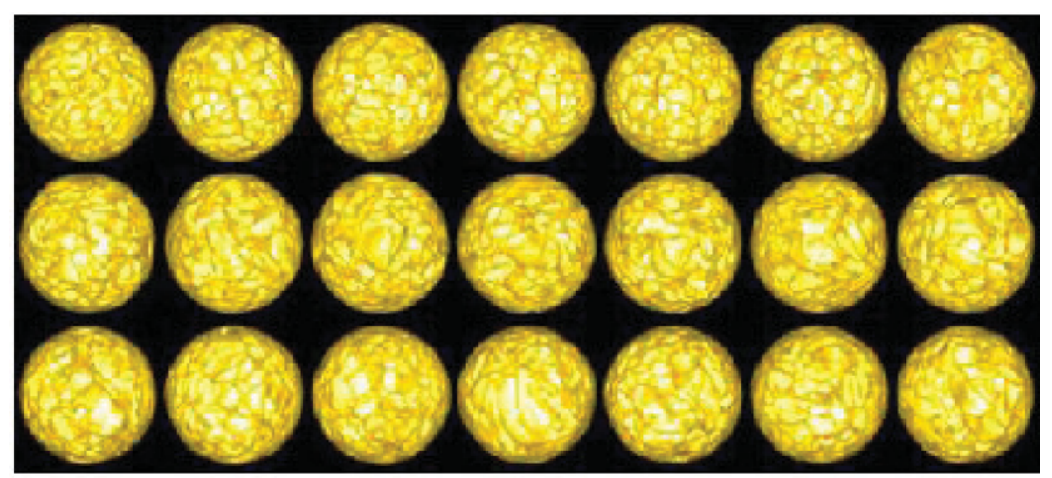

Figure 4. Simulations of global convection in a rotating reference frame from Steffen \& Freytag (2007) showing a 3 hour time series of the appearance of the photosphere. The idealized model consists of a scaled-down Sun with parameters similar to those of a red supergiant, but here with a rigid rotating core. The top row is a non-rotating calculation. The middle row displays the star viewed at the pole. The lower row represents the star as viewed at the equator. Large scale structures develop in the photosphere in the rotating model.

star with a rigidly rotating core by assuming a ratio of convective scale to stellar radius similar to a supergiant, shows the presence of large scale features (Fig. 4). If the model is made fully convective (as in a real supergiant) the meridional flow on the photospheric surface moves poleward above $60^{\circ}$ latitude and towards the equator at lower latitudes. All of these calculations assume a temperature structure that decreases outwards; there is no additional heating to produce a chromosphere. Thus while comparison to the infrared imaging observations is valid, it is not appropriate to use these models to interpret ultraviolet observations. High resolution spectroscopy of Betelgeuse in photospheric absorption lines (Gray 2008) suggests motions consistent with convection. The depths of neutral lines (V I, Fe I, and Ti I) show a pattern as a function of line core velocity that can be interpreted an increase in temperature of a volume of gas followed by an outflow (rising of hot cells), then followed by cooling and a falling back of the cooler material.

We need to be mindful that the oft-cited conjecture of Schwarzschild (1975) as confirmation of convective cells on the photosphere of a supergiant, really does not apply in detail as Uitenbroek et al. (1998) emphasized. The scalings used by Schwarzschild predicted 90 giant convective cells on a supergiant at one time, with a lifetime of 150 to 2000 days. In fact, photospheric observations (and the simulations) generally show 1-3 bright spots with lifetimes of 21-90 days. (Energy transport by convection stops at the photosphere, so this provides no insight into the upper layers in the chromosphere.) Numerical simulations currently provide the best support for convection as the source of photospheric spots.

\section{So what are the spots on Betelgeuse?}

In the photosphere (measured via the optical and infrared spectral regions), the large bright structures appear to be caused by convective motions, and these can be reproduced through a variety of radiative hydrodynamic simulations. Photospheric lines do not exhibit a predominant outflow, thus the convective cell motions do not initiate a mass outflow from the photosphere.

To understand the bright hot regions in the chromosphere, a calculation by Asida \& Tuchman (1995) is relevant. They argued that convective photospheric motions and pulsations in the photosphere would generate shock waves in the chromosphere and act 
to extend the upper atmosphere, facilitating outflow. And a steady warm outflow occurs at the level of the chromospheric Mg II k line (Uitenbroek et al. 1998). Rotation causes preferential break-out of the shockwave near the stellar pole. This occurs because rotation of an extended atmosphere causes shock waves traveling towards the pole to encounter a shorter path length and a steeper density gradient than in the equatorial direction. The amplitude of the wave towards the pole is larger, making the break-out region appear brighter. The discovery of a longitudinal magnetic field on Betelgeuse (Auriére et al. 2010) of $\sim 1$ G (similar to the Sun-as-a-star value) could aid in the channeling of ionized gas, in addition to offering the possibility of Alfvén wave acceleration and heating. The warm $(8000 \mathrm{~K})$ wind signaled by the outflowing Mg II k line may be driven by waves too.

\section{References}

Asida, S. M. \& Tuchman, Y. 1995, Astrophys. J., 455, 286

Auriére, M., Donati, J.-F., Konstantinova-Antova, R., Perrin, G., Petit, P., \& Roudier, T. 2010, Astron. Astrophys, 516, L2

Chiavassa, A., et al. 2010, Astron. Astrophys, 515, A12

Chiavassa, A., Plez, B., Josselin, E., \& Freytag, B. 2009, Astron. Astrophys, 506, 1351

Dupree, A. K., Baliunas, S. L., Guinan, E. F., Hartmann, L., Nassiopoulos, G., \& Sonneborn, G. 1987, Astrophys. J., 317, L85

Dupree, A. K., Lobel, A., \& Stefanik, R. 2010, to be submitted.

Dupree, A. K., Lobel, A., Young, P. R., Ake, T. B., Linsky, J. L., \& Redfield, S. 2005, Astrophys. J., 622,629

Gilliland, R. L. \& Dupree, A. K. 1996, Astrophys. J., 466, L29

Gray, D. F. 2008, AJ, 135, 1450

Harper, G. M. \& Brown, A. 2003, in: N. E. Piskunov, W. W. Weiss, \& D. F. Gray (eds.), Modelling of Stellar Atmospheres, Proc. IAU Symposium No. 210 (San Francisco: ASP), CDROM, F11

Harper, G. M., Brown, A., \& Guinan, E. F. 2008, AJ, 135, 1430

Harper, G. M., Brown, A., \& Lim, J. 2001, Astrophys. J., 531, 1073

Haubois, X. et al. 2009, Astron. Astrophys, 508, 923

Hebden, J. C. et al. 1986, Astrophys. J., 309, 745

Kervella, P. et al. 2009, A\&SA, 504, 115

Lim, J., Carilli, C. L., White, S. M., Beasley, A. J., \& Marson, R. G. 1998, Nature, 392, 575

Lobel, A. \& Dupree, A. K. 2000, Astrophys. J., 545, 454

Lobel, A. \& Dupree, A. K. 2001, Astrophys. J., 558, 815

Lovy, D., Maeder, A., Noels, A., \& Gabriel, M. 1984, Astron. Astrophys, 133, 307

Ohnaka, K., et al. 2009, Astron. Astrophys, 503, 183

Schwarzschild, M. 1975, Astrophys. J., 195, 137

Smith, M. A., Patten, B. M., \& Goldberg, L. 1989, AJ, 98, 2233

Steffen, M. \& Freytag, B. 2007, Astron. Nachr., 328, 1054

Stothers, R. \& Leung, K. C. 1971, Astron. Astrophys, 10, 290

Uitenbroek, H., Dupree, A. K., \& Gilliland, R. L. 1998, AJ, 116, 2501

\section{Discussion}

K. Strassmeier: I'm a little bit confused about a rotation period now. What is the rotation period?

DupreE: Yes, 17 years. That is a high value if you consider that Betelgeuse was about 15 solar masses on the main sequence. Assuming that it spins down as it evolves and becomes larger, a period of 17 years is a fast rotation. However, there may be reasons for this fast rotation. Theorists are inventive, and there have been suggestions that Betelgeuse may have swallowed its planets, so that the angular momentum of the star increased. 
Remember that Betelgeuse, as far as we know, is a single star. There is no confirmed companion. Calculations of stellar structure have been made, and it may be possible to have changes in the interior that would speed up the star. Another possibility is that we have an uncertainty of perhaps a factor of two in our measurement. So maybe the period is longer, but currently it appears a little fast.

MARK GiAmpapa: Are there observations of bisectors or inverse C shapes and of the amplitude?

DupreE: Yes. David Gray has a paper in 2008 where he did that, and he contends that with his bisectors he can identify rising expanding convective cells in the photosphere and so that he - he would confirm what I have been saying about infrared observations of the infrared imaging. It's Gray 2008, and he has done that, and he does it very carefully. He does confirm that and does think he sees the signatures of hot rising elements and cool falling elements in his C. This is measuring bisectors of photospheric lines. You need the signal to noise of 1,000, and you need resolution of a 100,000, and you can measure the profile very carefully and infer the velocity fields in the photosphere. 